\title{
A Review of DVR-based Routing Protocols for Mobile Ad Hoc Networks
}

\author{
Mrinal Kanti Debbarma \\ Research Scholar \\ Department of Information \\ Technology \\ Assam University, Silchar- \\ 788011, Assam, India
}

\author{
Santanu Kumar Sen \\ Professor, SMIEEE \\ Dept. of Computer Science \& \\ Engineering \\ Guru Nanak Institute of \\ Technology, Sodepur, Kolkata- \\ 700114
}

\author{
Sudipta Roy \\ Associate Professor \\ Department of Information \\ Technology \\ Assam University, Silchar- \\ 788011, Assam, India
}

\begin{abstract}
Designing an efficient and robust routing algorithm for Mobile Ad Hoc Network (MANET) is a challenging job compared to that of a wired and immobile network as because of some intrinsic characteristics of the MANET like highly dynamic nature of network topology due to mobility of nodes, asymmetric links, limited bandwidth, limited battery power, and alike. The statistics shows that the most of the routing algorithms, developed so far, for MANET, are conceptually based on the traditional distance vector routing algorithm which, in turn, is based on Distributed Asynchronous Bellman Ford's shortest path algorithm, however, with some minor to significant modifications as needed for the ad hoc and mobile nature of the MANET. A major thrust of the protocol designers towards the development and deployment of DVRbased routing protocols, irrespectively in wired, wireless or even in ad hoc networks, is primarily because of the conceptual and implementation simplicity and elegance coupled with the minimum information requirement by each node of the DVR. A good amount of research has been done in the past towards the improvement of routing algorithms in MANET, but the area has not become stable till date. This paper is presented towards the review of DVR-based routing protocols for finding path between source and destination in a mobile ad hoc network with significant comparisons on the characteristics, performance and complexity issues.
\end{abstract}

\section{Keywords}

DVR based routing protocols, Table-driven protocols, Ondemand routing protocols, Special neighbours.

\section{INTRODUCTION}

A MANET is a collection of mobile devices denoted as nodes, which can communicate between themselves using wireless links without the need or intervention of any infrastructure like base stations, access points etc [1][2][3]. A node in a MANET, which is equipped with a wireless transmitter and receiver (transceiver) and is powered by a battery, plays the dual role of a host and a router as well. Two nodes willing to communicate with each other need to be either in the direct common range of each other or should be assisted by other nodes acting as routers to carry forward the packets from a defined source to a destination in the best possible routing path [3,][4]. Designing routing protocol in MANET is quite difficult and tricky compared to that of any classic or non-ad hoc (formal) network due to some inherent limitations of the MANET like dynamic nature of network topology, limited bandwidth, asymmetric links, scalability, mobility of nodes limited battery power and alike. Moreover, the intrinsic nature of the nodes to move freely and independently in any arbitrary direction by potentially changing ones link to other's on a regular basis, is really an exigent concern while designing the desired routing algorithm. MANET is IP based and the nodes have to be configured with a free IP address not only to send and receive messages, but also to act as router to forward traffic to some destination unrelated to its own use.

The main challenge to setup a MANET is that each node has to maintain the information required to route traffic properly and thus designing a routing protocol for MANET has several difficulties. Firstly, MANET has a dynamically changing topology as the nodes are mobile. However, this behavior favors routing protocols that dynamically discover routes (e.g. Dynamic Source Routing [5], TORA [6], Associativity Based Routing (ABR) [7] etc.) over conventional distance vector routing protocols (DVR) [5][6][8]. Secondly, the fact that MANET lacks any structure and thus makes IP subnetting inefficient. Thirdly, limitation of battery power and power depletion of nodes due to large number of messages passed during cluster formation. Links in mobile networks could be asymmetric at times. If a routing protocol relies only on bidirectional links, the size and connectivity of the network may be severely limited; in other words, a protocol that makes use of unidirectional links can significantly reduce network partitions and improve routing performance.

\section{Classification of MANET Routing Protocols}

A large number of different routing protocols for MANET are either in use or have been suggested in the literature and the Figure 1 highlights some of them in terms of usability and importance in the specified field. These routing protocols can be classified on the basis of, what routing information is exchanged; when and how the routing information is exchanged; when and how routes are computed; and so on. Some classifications are as follows [7][8][9][10]:

(a) Link state routing vs. distance vector routing

(b) Pre-computed routing vs. on-demand routing

(c) Periodical update vs. event-driven update

(d) Flat structure vs. hierarchical structure

(e) Decentralized computation vs. distributed Computation

(f) Source routing vs. hop-by-hop routing

(g) Single path vs. multiple paths

A comparative discussion of the advantages and disadvantage between the two rival approaches in each class is presented below. 


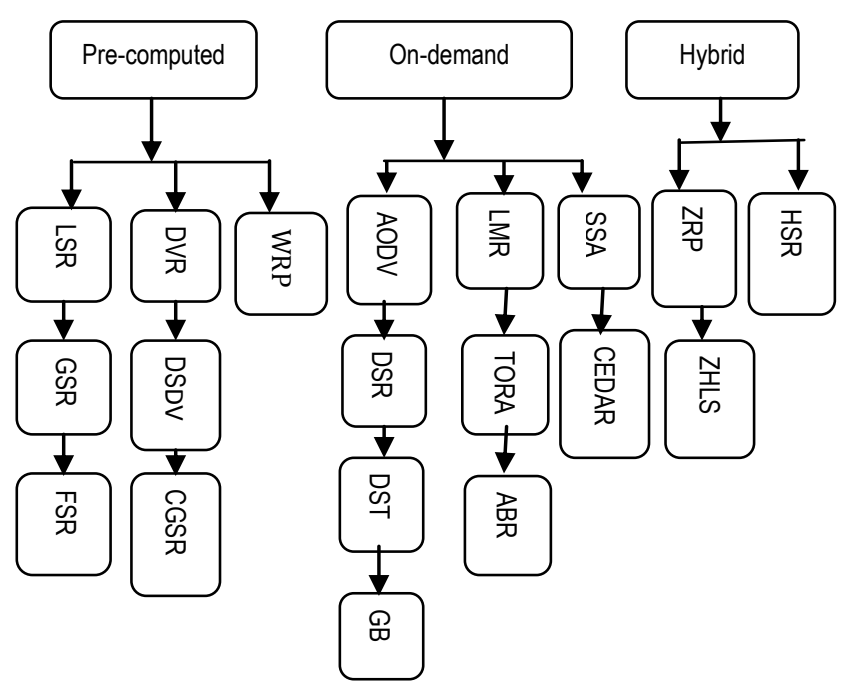

Figure 1: Classification of routing protocols in ad hoc network

\section{Link state routing (LSR) vs. distance vector routing} (DVR)

In Link state routing (LSR), routing information is flooded in the network by each router, in the form of link vectors. Any link change is flooded by the concerned routers into the entire network immediately. Every node can construct and maintain a global network topology when it receives this link vector from every other router and computes the length of its shortest path to each destination and stores this information in its routing table along with the identity of the neighbour, which provides the first path in the shortest path. LSR leads to very high control overhead because the ideology of the ad hoc networks is very dynamic and every change in the link takes a flood in the network.

In DVR, every node periodically exchanges distance vectors, containing its shortest path to every router in the network with its neighbors. When a node receives distance vectors from all its neighbors, it computes new routes (current shortest path) to every router and updates its distance vector accordingly. Every node reaches a remote destination via a neighbour which is acting as a forwarding node for that destination in its routing table. The major drawback of DVR is count-toinfinity problem which usually occurs followed by a link failure or router failure. The other problems of DVR are slow convergence, temporary routing loops and route oscillations.

(b) Pre-computed routing vs. on-demand routing (Proactive vs. Reactive)

Depending on when the route is computed, routing protocols can be divided into two categories: pre-computed routing and on-demand routing.

Pre-computed routing is also called proactive routing or tabledriven routing. In this approach, the routes to all destinations are computed a priori. In order to compute routes in advance, nodes need to store the entire or partial information about link states and network topology. In order to keep the information up to date, nodes need to update their information periodically or whenever the link state or network topology changes. The advantage of pre-computed routing is that when a source needs to send packets to a destination, the route is already available, i.e. there is no route discovery delay. The disadvantage is that some routes may never be used, although the dissemination of routing information consumes a lot of the scarce wireless network bandwidth and node battery power when the link state and network topology change fast (this is especially true in a wireless ad hoc network). The conventional LSR and DVR are examples of proactive routing.

On-demand routing is also called reactive routing. In this method, the route to a destination may not exist in the routing table in advance and it is computed only when the route is needed.

Route discovery: When a source needs to send packets to a destination, first it finds a route or several routes to the destination. This process is called route discovery.

Route maintenance: After the route is discovered, the source transmits packets along the route. During the transmission of packets, the route may be broken because the node on the route moves away or goes down. The broken route needs to be rebuilt. The process of detecting route breakage and rebuilding the route is called route maintenance. The major advantage of on-demand routing is that the precious bandwidth of wireless ad hoc networks is greatly saved because it limits the amount of bandwidth consumed in exchange of routing information by maintaining routes to only those destinations to which the routers need to forward data. On-demand routing removes the need for disseminating routing information periodically. The primary problem with on-demand routing is the large latency at the beginning of the transmission caused by route discovery.

Finally, the hybrid protocols try to combine the both proactive and reactive protocols and also take advantage of the availability at low cost of the Global Positioning System (GPS) [11], for achieving improved performance through location dependent routing.

(c) Periodical update vs. event-driven update

Periodical update protocols disseminate routing information periodically. Periodical updates will simplify protocols and maintain network stability, and most importantly, enable (new) nodes to learn about the topology and the state of the network. However, if the period between updates is large, the protocol may not keep the information up-to-date. On the other hand, if the period is small, too many routing will be disseminated which would waste the precious bandwidth of a wireless network.

In an event-driven update protocol, when events occur (such as when a link or a new link appears), an update packet will be broadcast and the up-to-date status can be disseminated over the network soon. The problem might be that if the topology of networks changes rapidly, a lot of update packets will be generated and disseminated over the network which will use up a lot of precious bandwidth, and furthermore, may cause too much fluctuation of routes. One solution is to use some threshold.

Periodical update and event-driven update mechanism can be used together, forming what is called a hybrid update mechanism. For example, in Destination Sequence Distance Vector routing protocol (DSDV) [10][12], a node broadcasts its distance-vector periodically. Moreover, whenever a node finds that a link is broken, it distributes a message immediately.

\section{(d) Flat structure vs. hierarchical structure}

In a flat structure, all nodes in a network are at the same level and have the routing functionality. Flat routing is simple and efficient for small networks. The problem is that when a 
network becomes large, the volume of routing information will be large and it will take a long time for routing information to arrive at remote nodes.

For large networks, hierarchical (cluster-based) routing may be used to solve the above problems. In hierarchical routing, the nodes in the network are dynamically, organized into partitions called clusters. The clusters are aggregated again into larger partitions called super-clusters and so on. Organizing a network into clusters maintain a relatively stable network topology. The high dynamics of membership and network topology is limited within clusters. Only stable and high level information such as the cluster level or the supercluster level will be propagated across a long distance, thus the control traffic (or routing overhead) may be largely reduced. Within a cluster, the nodes may have complete topology information about its cluster and proactive routing may be used. If the destination is in a different cluster from the source, inter-cluster routing must be used. Inter-cluster routing is generally reactive, or a combination of proactive and reactive routing. Similar to cellular structure in cellular systems, a hierarchical cluster is readily deployable to achieve some kind of resource reuse such as frequency reuse and code reuse and interference can be reduced when using different spreading codes across clusters.

(e)

$$
\begin{aligned}
& \text { Decentralized computation vs. distributed } \\
& \text { Computation }
\end{aligned}
$$

In a decentralized computation-based protocol, every node in the network maintains global and complete information about the network topology such that the node can compute the route to a destination itself when desired. The route computation in LSR is a typical example of decentralized computation.

In a distributed computation-based protocol, every node in the network only maintains partial and local information about the network topology. When a route needs to be computed, many nodes collaborate to compute the route. The route computation in DVR and the route discovery in on-demand routing belong to this category.

\section{(f) Source routing vs. hop-by-hop routing}

Some routing protocols place the entire route (i.e., nodes in the route) in the headers of data packets so that the intermediate nodes simply forward these packets according to the specified route in the header. Such a routing is called "source routing". Source routing has the advantage that intermediate nodes do not need to maintain up-to-date routing information in order to route the packets they forward, as the packets themselves already contain all the routing decisions. This fact, when coupled with on-demand route computation, eliminates the need for the periodic route and neighbor detection. The biggest problem with source routing is that when the network is large and the route is long, placing the entire route in the header of every packet will waste a lot of scarce bandwidth.

In hop-by-hop routing, the route to a destination is distributed in the "next hop" of the nodes along the route. When a node receives a packet for a destination, it forwards the packet to the next hop corresponding to the destination. The problems are that all nodes need to maintain routing information and there may be a possibility of forming a routing loop. (g) Single path vs. multiple paths

Some routing protocols find a single route from a source to a destination, which results in simple protocol and saves storage, whereas other routing protocols find multiple routes, which have the advantages of easy recovery from a route failure and thus making routing algorithm reliable and robust. Moreover, the source cannot only select the best one among multiple available routes, but also distribute the load among them thus presenting possible congestion in the network.

\section{Review of some popular Routing Algorithms}

Broadly, the routing algorithm in MANET has been classified into three categories, viz. proactive or table-driven, reactive or on-demand and the hybrid. The dominant algorithm used in the wired network are proactive in nature but some of the features of a MANET make a reactive approach to perform better under many situations. Because of this observation, hybrid approach is gaining more importance.

In this section, three popular routing protocols are reviewed for MANETs winch are primarily based on proactive or reactive approach. The first one is most well-known proactive protocol, the other two are reactive in nature and, additionally one of the reactive protocols employ source routing for MANET.

\subsection{Table-driven or Proactive protocols}

Proactive or table-driven protocols are based on one of the old techniques of routing. These protocols maintain route to all the nodes present in the network, all the time. To ensure the freshness of the routing tables, these protocols adopt different mechanisms, e.g., sending the "hello" messages containing the address of transmitting node, at regular intervals. Some of the popular table-driven routing protocols (proactive) for MANETs are listed below: Destination Sequence Distance Vector routing

(a) Destination Sequence Distance Vector (DSDV) [10][12]

(b) Wireless Routing Protocol (WRP) [13]

(c) Cluster-head Gateway Switch Routing (CGSR) [10]

(d) Optimized Link State Routing protocol (OLSR) [14]

(e) Fisheye State Routing protocol (FSR) [15][16]

(f) Global State Routing protocol (GSR) [17]

(g) Topology broadcast based on Reverse-Path Forwarding routing protocol (TBRBF) [2][10]

(a) Destination Sequence Distance Vector (DSDV)

DSDV is the most well-known proactive routing scheme. It is based on the classical Distributed Asynchronous Bellman Ford (DBF) routing algorithm [9], which has long been used in for wired networks. DSDV is a table driven routing algorithm and maintains routing tables containing route for each station present in the network.

DSDV uses the sequence number to keep the route fresh and to avoid the loop. Each entry in the routing table must contain, destination id, destination number, the next hop for this destination. The sequence number is associated with each node, which increases monotonically. The information with sequence number is treated as fresh information.

The nodes exchange routing updates whenever they detect a change in topology. Two types of updates are used in DSDV, namely, full dumped and incremental update. In case of full updates, the complete routing table is sent out and in case of an incremental or partial updates only the changes. When the 
network is relatively stable, incremental updates are sent to avoid extra traffic and full dumps are relatively infrequent. In a fast-changing network, incremental packets can grow big so full dumps will be more frequent.

\section{(b) Wireless Routing Protocol (WRP)}

The WRP protocol [8] also guarantees loops freedom and it avoids temporary routing loops by using the predecessor information. However, four routing tables are required in each node in WRP protocol. This presents a significant amount of memory overhead at every node as the network size increases. Another disadvantage of WRP is that it ensures connectivity through the use of hello message.

\section{(c) Cluster-head Gateway Switch Routing (CGSR)}

A cluster-based routing protocol is the most popular hierarchical routing strategy. It uses a particular clustering algorithm for election of cluster head in which mobile nodes are grouped into clusters by geographic vicinity. Cluster heads look into all responsibility on behalf of the cluster for membership management and routing functions. CGSR [18] is an example of a cluster-based MANET routing protocol. The Hierarchical State Routing (HSR) protocol [19] also supports a multi-level cluster arrangement. CGSR protocol differs from the previous protocol in the type of addressing and in the employment of network group structure. Instead of a "flat" network, CGSR is based on a clustered multihop mobile wireless network with several heuristic routing structures

$$
\text { Optimized Link State Routing Protocol (OLSR) }
$$

OLSR is a point-to-point routing protocol based on the traditional LSR algorithm. In this scheme, every node maintains topology information about the network periodically replacing link-state messages. The originality of OLSR is that it minimizes the size of each control message and the number of rebroadcasting nodes during each route update by employing multipoint replaying (MPR) approach. During each topology update, each node in the particular network selects a set of intermediate/neighbouring nodes to retransmit/forward its own packets. This set of nodes is termed as the multipoint relays of that node. Any node which is not in the set can read and process each packet but do not retransmit. To select the MPRs, each node periodically broadcasts a list of its one hop neighbours using hello messages from the list of nodes in the network.

$$
\text { Fisheye State Routing protocol (FSR) }
$$

FSR decreases the size of the update messages in GSR by updating the network information neighbour nodes at a higher frequency than for the isolated nodes, which lie outside the fisheye scope. This makes FSR more scalable to dense networks than the protocols described. However, scalability originates at the price of reduced accuracy. As mobility grows the routes to remote destination become less accurate. This can be overcome by making the frequency at which updates are sent to remote destinations proportionate to the level of mobility.

\section{(f) Global State Routing protocol (GSR)}

GSR protocol is based on the traditional LSR algorithm. However, GSR has upgraded the way information is disseminated in LSR algorithm by restricting the update messages between intermediate nodes. In GSR, every node maintains a link state table (LST) based on the up-to-date information received from neighbouring nodes, and periodically interchanges its link state information with neighbouring nodes. However, the size of update messages is relatively dense, and as the size of the network increases they will get even larger. Therefore, a substantial amount of bandwidth is consumed by these update messages.

\section{(g) Topology broadcast based on Reverse-Path} Forwarding routing protocol (TBRBF)

TBRPF protocol is based on link-state based routing protocol, which implements hop-by-hop routing scheme. Reverse-path forwarding (RPF) is used to propagate its update packets information in the opposite direction along the spanning tree, which is made up of the minimum-hop path from the nodes leading to the source of the update message. In this routing approach, every node computes a source tree, by providing a path to all reachable destinations by applying a modified version of Dijkstra's algorithm (MDA) [5][8] on the partial topology information stored in their topology table. In TBRPF, every node minimizes overhead by reporting only part of their source tree to their neighbours not all the nodes. The reportable part of each source tree is interchanged with neighbouring nodes periodically along with hello messages.

\subsection{On-demand or Reactive routing protocols}

The reactive routing protocols discover the route(s) to destination(s) only when it is needed. When a node requires a route to destination, it initiates route discovery process within the network. After getting some routes to the destination, the best among them is selected and used for forwarding the packets to the destination. Once a route is discovered, it is maintained by route maintenance procedure until either destination becomes inaccessible along every path from source or the route is no longer desired. Below is the list of some Reactive ad hoc routing protocols.

(a) Ant-based Routing Algorithm (ARA) [11]

(b) Associativity-Based Routing (ABR) [7][20]

(c) Ad hoc On Demand Distance Vector (AODV) [15][21][22]

(d) Dynamic Source Routing protocol (DSR) [10][23]

(a) Ant-based Routing Algorithm for mobile ad hoc Networks (ARA)

This protocol is based on swarm intelligence and ant colony based meta heuristic search. The ARA for ad hoc network attempts to reduce routing overheads by adopting the food searching techniques (FST) of ants. During ants search for food, they start from their nest and walk in the direction of the food, while leaving behind a transient trail called pheromone. This directed the path that has been taken by the ant and allows rest to follow, until the pheromone disappears. Similar to AODV and DSR, ARA is also made up of two phases:

\section{- $\quad$ Route discovery \\ - Route maintenance}

During route discovery forwarding ANT (FANT) is broadcast through the network (like a RREQ). At each hop, each node calculates total pheromone value depending on how many number of hops the FANT has taken to reach them. The nodes then forward the FANT to their neighbours. Once the destination is reached, it creates a Backward ANT (BANT), and returns to the source. When the source receives the BANT from the destination node, a path is determined and data packet propagation begins. For maintaining each route, every time a data packet travels between forwarding nodes and the pheromone value is increased. Otherwise, the pheromone value is decreased overtime until it expires. Making a broken 
link, the nodes check their routing table, if no route is found they inform their intermediate neighbours for a substitute route. If the neighbours have a route they inform their neighbours by backtracking. If the source node is reached and no route is found, a new route discovery process is initiated.

Advantages: The advantage of these techniques is that the size of each FANT and BANT is tiny, which means the amount of overhead per control packet introduced in the network is minimized. However, flooding is used for route discovery, which means that the protocol may have scalability problems as the number of nodes and flows in the network grows.

\section{(b) Associativity Based Routing protocol (ABR)}

ABR protocol defines a new type of routing metric "degree of association stability" for MANETs. In this routing protocol, a selection of route is based on the degree of association stability of mobile nodes. Each node periodically generates beacon to broadcast its presence. Upon receiving the beacon message, a neighbor node updates its own associativity table. For each beacon received, the associativity tick of the receiving node with the beaconing node is increased. A high value of associativity tick for any particular beaconing node means that the node is relatively static. When any neighboring node moves out of the neighborhood then associativity tick is reset.

\section{(c)Ad hoc On-Demand Distance Vector Routing Protocol} (AODV)

AODV is another reactive routing protocol, uses sequence number to avoid looping and maintains fresh routes to the destinations. This algorithm also consists of two phases, route discovery and route maintenance.

Route discovery: If the route to the destination is not available in the routing table, a RREQ (Route Request) packet is broadcast throughout the MANET with a search ring technique. When a node receives this RREQ, the node creates a reverse routing entry towards the originator of RREQ, which is used to forward replies later. The destination or the intermediate node, which has a valid route towards the destination, answers with a RREP (Route Reply) unicast packet. On receipt of the RREP the reverse routing entry towards the originator of RREP is also created, the processing of RREQ. A precursor list is maintained to notify upstream about the link failure of the downstream neighbour for a particular destination.

Route maintenance: Every node along an active route periodically broadcasts messages to its neighbours. If the node does not receive a HELLO message or data packet from a neighbour for a while, the link between itself and the neighbour is to be broken. RERR (Route Error) packet is sent to the neighbours in the list associated with the routing entry to inform them of the link failure. AODV gives all round good performance in small and medium size networks.

It is interesting to note that most of the successful and popular routing protocols like DSDV, AODV, ADV [24] etc. used in MANETs are based on the fundamental principle of distance vector routing (DVR), which in turn, derived from the wellknown Distributed Asynchronous Bellman Ford Algorithm.

\section{(d) Dynamic Source Routing Protocol (DSR)}

DSR is a source routing protocol and behaves in a reactive manner for discovering the route to a destination. It does not maintain the routing table like other routing protocols. DSR has a route cache, where complete path (list of all intermediate nodes) to the destination is kept. The basic procedure of DSR is divided two phases:

Route discovery: If the source route entry towards a destination is not present in the route cache, a Route Request packet is broadcast throughout the MANET. Every receiving node, if it has the route to the requested destination, adds its id in the list in the request packet's header. When the destination receives the packet, it also discovers the route to reach the source in the similar way.

Route maintenance: Unlike the proactive routing protocols and the AODV, no HELLO message is sent in the DSR. Every node along the path is responsible for checking the validity of the downstream link connecting itself and the next hop in the source route, which could be detected by its MAC layer. If link breakage is found, the source of the route will be notified with a Route Error packet. The source then reinitiates a route discovery procedure.

Route cache is very helpful in reducing the control overhead in DSR. Gratuitous reply, multi-paths and packet salvaging are the features that take help from cache. During route discovery phase, if the intermediate node has the route towards the destination in its routing cache, it can answer with a route reply packet and send a gratuitous route reply about the source to the destination at the same time.

Multiple paths are available by the route discovery process. When an intermediate node detects the downstream link breakage when forwarding a data packet, but it has another source route in its routing cache towards the same destination, it forwards the packet along the new route, which is called packet salvaging.

\subsection{DVR based Routing protocols in MANETS}

DVR is a simple, elegant and widely used as the Internet Gateway Protocol (IGP) and Extended Gateway Protocol (EGP) [8] in the Global Internet as well as in the private Internets in the form of Routing Information Protocol (RIP)[8][25], RIP-2 and Boarder Gateway Protocol (BGP)[8][25]. The Distance Vector Routing Algorithm (DVRA) is a dynamic, distributed, asynchronous and iterative routing protocol where the routing tables are continuously updated with the information received from the neighbouring routers [9][25].

As a distributed dynamic routing algorithm which is expected to adapt to the changes in topology and traffic. The existing DVRA, though simple and conceptually elegant, suffers from some well-known problems like Count-to-Infirmity (CTI), slow convenience, looping, frequent route oscillation. Out of all these, the CTI is more vulnerable, which actually, made practical DVR out of race [26].

Though good amount of research has been done in the past towards the improvement of the basic DVRA, none of the proposed techniques like Hold-Down, Split Horizon and Poison Reverse have been able to solve the problems in the DVRA satisfactorily.

DVRA is used in routing protocols similar to IGRP [27], ISO and Novell IPX due to its simplicity, elegance and flexibility.

A modified DVRA (MDVRA) [26] is investigated, which uses a host of novel techniques to remove all the weaknesses, particularly the CTIP, inherent to the existing DVRA. Additionally, the goal is to augment the existing capabilities 
of the DVRA so as to make it efficient, robust, fully dynamic and fully fit to the environment of the MANET primarily and WSN in the extended phase.

\subsection{Some Problems of DVRA}

There are some weaknesses of the asynchronous DVRA are as follows:

- Count-To-Infinity (CTI) Problem

- Slow Convergence Problem

\subsubsection{Count-To-Infinity Problem (CTIP)}

The DVRA cannot perform fine if there are topological changes in the internetwork. DVRA suffers from CTI problem due to link or router failure or due to unending routing loops. The CTI caused by routing loops involving more than two routers are providing in [28].

\subsubsection{Slow Convergence Problem (SCP)}

When there is growth in terms of cost of any link or there is a failure in the link between two corresponding neighboring nodes in an internetwork, then it is considered as worst case or requires unnecessary number of iterations to terminate.

\subsection{Modification of the DVRA}

There are some key modifications ideas from the MDVRA have been developed in the design of the MDVRMANET [26][28]. How these special neighbours are identified, primarily by using the DVRTs and how they are used towards making the MDVRA efficient and practical routing algorithm with fast convergence and no CTI problem.

A) The first essential modification requires that each entry in the DV Tables (DVT), periodically exchanged between the neighbours in a DVRA, should include the next-hop identity for the route. This information is not included in the DVTs in the DVRA but is contained in every router's own DVR Table (DVRT).

B) The second modification requires a router to perform in a more intelligent and proactive manner in trying to determine the alternative shortest path following a link failure.

C) Third modification requires each router to identify some of its neighbours as special neighbours and use them for avoiding routing loops and/or achieving a very reliable and efficient node-to node communication. The concept of the special neighbours and the benefits a node derives from each of them are as follows:

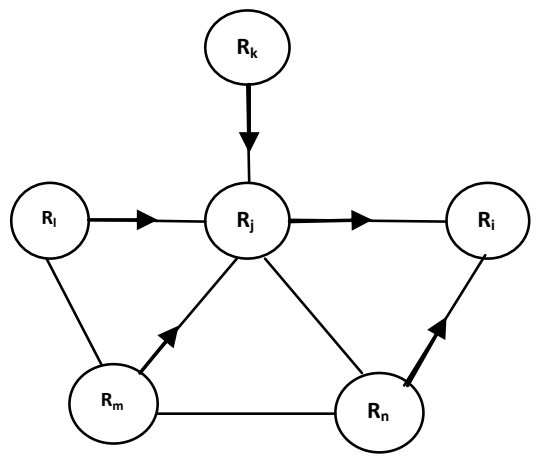

Figure 2: An Example network graph for illustrating concepts of some special neighbours. (a) Forwarding Neighbour (FN): If a router $R_{j}$ presently reaches a non-neighbour or remote destination $R_{i}$ via its neighbour $R_{k}$ (because $R_{k}$ provides $R_{j}$ with the current shortest path to reach $R_{i}$ ), then $R_{k}$ is the FN of $R_{j}$ for reaching Ri. In Figure $2, R_{j}$ is the $F N$ of $R_{k}, R_{1}$ and $R_{m}$ (but not of $R_{n}$ ) for reaching $R_{\mathrm{i}}$. In the MDVRA, an explicit distinction is made between a Nh neighbour and a FN. Since a neighbour is always reached directly, i.e., never via another neighbour, each neighbour in the MDVRA is always its Nh neighbour. But a neighbour is a FN only when it is used to forward packets to a remote or non-neighbour destination. Thus, in the MDVRA, all neighbours are the Nh neighbours for reaching themselves but all of them may not be FNs.

(b) Dependent Neighbour (DN): If a router $R_{k}$ presently reaches a destination $R_{i}$ via its neighbour $R_{j}$, i.e., if $R_{k}$ depends on $R_{j}$ for reaching $R_{i}$, then $R_{k}$ is a Dependent Neighbour (DN) of $R_{j}$ for reaching $R_{i}$. The routers $R_{k}, R_{l}$, and $R_{m}$, but not $R_{n}$, in Figure 2 are all DNs of $R_{j}$ for reaching the destination $\mathrm{R}_{\mathrm{i}}$. Awareness about its $\mathrm{DN}(\mathrm{s})$ for each destination is extremely important to the router y because of two reasons. First, a DN x can never provide it with a shorter path to reach $\mathrm{z}$ so that the advertisements of the $\mathrm{DN} \mathrm{x}$ for the destination $\mathrm{z}$ should just be ignored. Second, if y ever loses its present path (route) to $\mathrm{z}$ and looks for the shortest path to reach $\mathrm{z}$, choosing the DN $\mathrm{x}$ as the next-hop must be avoided because that will create a routing loop between $\mathrm{x}$ and $\mathrm{y}$.

(c) Co-Neighbour (CN): If three routers $\mathrm{R}_{\mathrm{j}}, \mathrm{R}_{\mathrm{k}}$ and $\mathrm{R}_{\mathrm{l}}$, form a triangle in a network graph, then any two of the three routers are mutual CNs of each other for the third one, e.g., in Figure 2, $R_{k}$ is a CN of $R_{j}$ for $R_{l}, R_{l}$ is a $C N$ of $R_{j}$ for $R_{k}, R_{j}$ is a $C N$ of $R_{k}$ for $R_{1}$, and so on. Again for the router $R_{j}, R_{k}$ has no $C N$, $R_{l}$ has one $C N$, namely, $R_{m}, R_{m}$ has two CNs, namely, $R_{l}$ and $R_{n}, R_{n}$ has two CNs, namely, $R_{m}$ and $R_{i}$ and, finally, $R_{i}$ has one $\mathrm{CN}$, namely, $\mathrm{R}_{\mathrm{n}}$.

(d) Co-Dependent Neighbour (CDN): If routers $R_{k}$ and $R_{1}$ are CNs of each other (i.e. mutual CNs) for the router $R_{j}$ and, additionally, both $R_{k}$ and $R_{l}$ are also DNs of $R_{j}$ for reaching a common destination $R_{i}$, i.e., $R_{j}$ is their common $F N$ for reaching $R_{i}$, then $R_{k}$ and $R_{l}$ are CDNs of $R_{j}$ for the destination $R_{i}$. In accordance with the arrows shown in Figure $2, R_{1}$ and $R_{m}$ are CDNs of $R_{j}$ for reaching Ri. Incidentally, it may be noted that $R_{n}$ would also have become, along with $R l$ and $R_{m}$, a CDN of $R_{j}$ for reaching $R_{i}$, had the link $R_{n} R_{i}$ been absent.

(e) Single-Connected Neighbour (SCN), Pendant Neighbour (PN), Sole Neighbour (SN) and Lost Destination (LD):

If $y$ is the only neighbour of $\mathrm{x}$, then $\mathrm{y}$ is the $\mathrm{SN}$ of $\mathrm{x}$ and $\mathrm{x}$ is a PN of $y$. A node in the network graph having a degree 1, i.e., a router which is connected only to a single router, is called a Single-Connected Neighbour (SCN) of the sole router to which it is connected. The sole router recognizes its $\mathrm{SCN}$ as a Pendant Node (PN) in the network. The router $\mathrm{R}_{\mathrm{k}}$ in Figure 2 is a $\mathrm{SCN}$ of $\mathrm{R}_{\mathrm{j}}$ and a PN in the network graph. It is obvious that a PN can only be a DN but never a FN. In case a SN loses communication with its $\mathrm{PN}$, it views the PN as a LD, does not look for an alternative route to reach it (till its direct communication with the PN is re-established) and informs all other neighbours about this LD having become unreachable. This ensures that no more packets are generated for or forwarded to the LD by any router. 


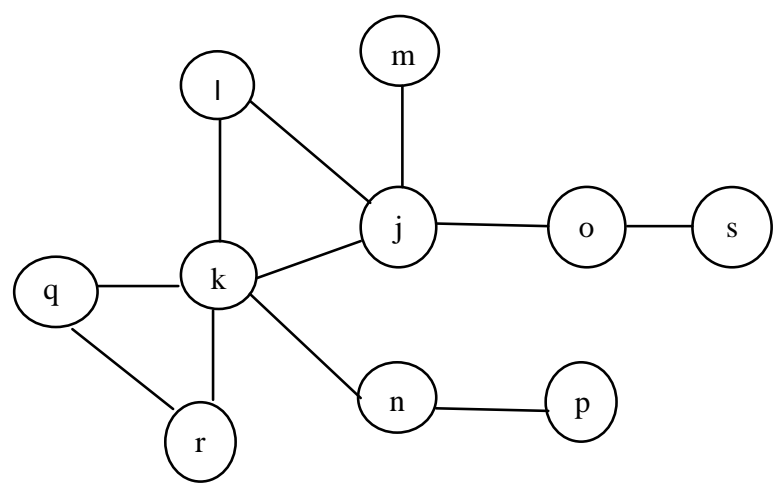

Figure: 3. Sample Network with special neighbours of Router j

A DVRT has $\mathrm{N}$ entries one corresponding to each known router in the $\mathrm{N}$-node network. Each entry has 3 fields, namely, the identity of a destination router, the estimated distance (metric) of this router and, finally, the identity of the next-hop (Nh) router, i.e., the $\mathrm{FN}$ in case of any remote router, for reaching that destination. In this paper we shall normally consider delay as the metric since the MDVRA is expected to be fully adaptive, i.e., adaptive in respect of both topology and traffic. The short-form notations $\mathrm{Des}_{\mathrm{i}}, \mathrm{Dis}_{\mathrm{i}}$ and $\mathrm{Nh}_{\mathrm{i}}$ will represent the values in the three fields of the $i$-th entry, $i=1,2$, 3, N in the DVRT of each router. The DVRT of the router $R_{j}$ will be denoted by DVRT $\mathrm{j}_{\mathrm{j}}$. It should be reiterated that the $\mathrm{Nh}_{\mathrm{i}}$ field will identify either the destination itself or the FN, depending upon the Des $s_{i}$ being a neighbour or a nonneighbour.

\begin{tabular}{|c|c|c|}
\hline Des & Dis & Nh \\
\hline $\mathrm{k}$ & $\mathrm{D}_{\mathrm{ik}}$ & $\mathrm{K}$ \\
\hline $\mathrm{l}$ & $\mathrm{D}_{\mathrm{il}}$ & $\mathrm{L}$ \\
\hline $\mathrm{m}$ & $\mathrm{D}_{\mathrm{im}}$ & $\mathrm{M}$ \\
\hline $\mathrm{n}$ & $\mathrm{D}_{\mathrm{in}}$ & $\mathrm{N}$ \\
\hline $\mathrm{o}$ & $\mathrm{D}_{\mathrm{io}}$ & $\mathrm{O}$ \\
\hline $\mathrm{p}$ & $\mathrm{D}_{\mathrm{ij}}$ & $\mathrm{N}$ \\
\hline $\mathrm{q}$ & $\mathrm{D}_{\mathrm{iq}}$ & $\mathrm{K}$ \\
\hline $\mathrm{r}$ & $\mathrm{D}_{\mathrm{ir}}$ & $\mathrm{K}$ \\
\hline $\mathrm{s}$ & $\mathrm{D}_{\mathrm{is}}$ & $\mathrm{O}$ \\
\hline
\end{tabular}

Table 1: Routing Table of Router $\mathbf{j}\left(\mathrm{DVRT}_{\mathbf{j}}\right)$

Notations used in network graph, routing table are Des: Destination, Dis: Distance, Nh: Next-hop router, DFC: distance between neighbor RF and destination RC., FN: Forwarding Neighbour, CN: Co-Neighbour, CDN: CoDependent Neighbour, SCN: Single Connected Neighbour, PN: Pendant Node, LD: Lost Destination, DVRT $_{j}$ : Distance Vector Routing Table of node j, Nbr: Neighbour, N: Total no. of router entries in DVRT $_{j}$ i.e., total no. of nodes in the network, $\mathrm{Nj}$ : Total no. of neighbours of $\mathrm{j}$.

\section{5 a. Identification and Use of the Neighbourhood}

A router in the MDVRA always remains well-aware about its current neighbourhood so as to be able to use all the information that may be collected through them. This specially requires the router to identify its various special neighbours, namely, FN, DN, CN, CDN, SCN, PN, SN, LD. Based on the above method of identifying and utilizing each of these special types of neighbours, to meet the two major challenges in designing routing algorithms for MANET, specifically, highly dynamic changes in the MANET topology and the need to preserve battery power, a novel idea of neighbourhood monitoring has been employed. It was identified that DVR is based on a neighbour-exchange algorithm; a proactive approach to dealing with neighbours is much more desirable in a MANET than reacting to the discovery gains of neighbours after they actually occur.

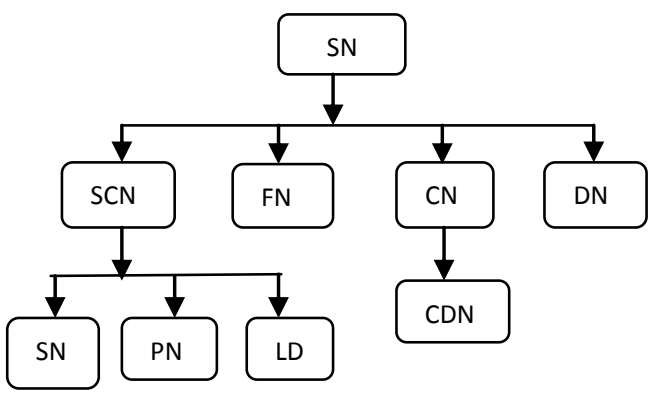

Figure 4: Interrelationship between different types special neighbours

\section{5 b. Neighbour Notification (NN) and DVRT Packets: Periodic Interchange}

DVRT periodically updates to enable each router, in an energy-aware manner, to both monitor its neighbours, the periodic DVRT exchange process in the DVRA has been divided in MDVRMANET into two separate exchanges, namely, more frequent exchange of Neighbour Notification (NN) packets and less frequent exchange of regular DVRT packets. Through the short NN packets, a node broadcasts to all neighbours its transmitted power as well as the list of its current RNs. DVRT packet contains all the information hold in an NN packet; it effectively replaces an NN packet. That is, during every 15 second neighbour exchange cycle, each router synchronously sends and asynchronously receives from each neighbour 4 consecutive NN packets monitored by a DVRT packet.

\section{Conclusions and Future Work}

The problems of routing in MANETs and other such ad hoc networks like wireless sensor networks (WSN), Vehicle Ad hoc Networks (VANET), etc are primarily because of the mobility of the nodes, random topology changes, limited battery power, limited bandwidth, asymmetric links, frequent link breakage due to random addition and deletion of nodes, to name a few, for which, many routing protocols already proposed and/or in research, have not found to be stable and thus providing enough scope of improvement through research and investigations.

In this paper, several issues, in the perspective of routing in MANET have been discussed and many routing schemes in the said field have been investigated and compared with each other keeping technical relevancy, as far as practicable. The review shows that a majority class of existing routing protocols is fundamentally DVR based as because of the conceptual and implementation simplicity and elegancy of the DVR. The neighbour dependency of the DVR protocol, which has become strength of the protocol, as a router could see its whole world only through its next-hop neighbours, has not only lowered the burden of preserving and maintaining the total network information, but also has reduced the burden of bandwidth and battery supply and alike overheads, and thus making the DVR or DVR-based algorithm more attractive and interesting. In this paper, a DVR-based routing algorithm for 
MANETs has been investigated and suggested keeping stress on the neighbour characteristics of the protocol. The key focus is made on neighbour categorization with proper identification, so that each neighbour can provide more and more information of different nature to its adjacent router and thus each router could enrich its information database by collecting such information from all of its next-hop neighbours, which would help during routing traffic proactively or reactively as the situation demands.

The future works will be focused towards the hybrid MANET protocols, as such protocol is in demand of late because of technical feasibility. The chief motivation towards the design of a hybrid MANET protocol would be towards the three practically vital issues, viz., implementation simplicity, robustness and efficiency.

\section{REFERENCES}

[1] R. Ramanathan and J. Redi, "A Brief Overview of Ad Hoc Networks: Challenges and Directions ", IEEE Communications, Volume 40, Issue 5, May 2002

[2] Simplot Ryl D., Stojmenovic I., "Guest Editorial - AdHoc Networking: Data Communications and Topology Control", IEEE Network, Volume 18, Issue 4, July 2004

[3] Jie Wu, Stojmenovic I., “Ad Hoc Networks", IEEE Computer, Volume 37, Issue 2, Feb 2004

[4] G.V. Kumar et al. "Current Research Work on Routing Protocols for MANET: A Literature Survey" International Journal on Computer Science and Engineering, Vol. 02, No. 03, 2012, 706-713

[5] Albeto Leon-Garcia and Indra Widjaja, Communication Networks, Tata McGraw Hill, 2000

[6] N. H. Saeed, M.F. Abbod, and H S. AI-Raweshidy, "MANET Routing Protocols Taxanomy", IEEE International Conference on Future Communication Networks, PP-123-128, 2012

[7] M. Abolhasan et al. "A Review of Routing Protocols for Mobile Ad Hoc Networks" Elsevier Ad Hoc Networks 2 (2004) 1-22

[8] A. S. Tenenbaum, Computer Networks, 3rd Ed., PHI, 2000

[9] D. Bertsekas and R. Gallagar: "Data Networks", 2nd Ed., Englewood cliffs, NJ, Prentice Hall, 1992

[10] E. M. Royer and C. K. Toh, "A Review of Current Routing Protocols for Ad Hoc Mobile Wireless Networks", IEEE Personal Communications, April 1999, pp. $46-55$

[11] M. Gunes, U. Sorges, I. Bouazizi, Ara-the ant-colony based routing algorithm for manets, in: ICPP Workshop on Ad Hoc Networks (IWAHN 2002), August 2002, pp. 79-85.

[12] C. E. Perkins and P. Bhagwat, "Highly dynamic Destination-Sequence Distance Vector Routing (DSDV) for Mobile Computers", Computer Communication Review, Oct 1994, pp. 234-244

[13] S. Murthy J.J. Garcia-Luna-Aceves, A routing protocol for packet radio networks, in: Proceedings of the First Annual ACM International Conference on Mobile Computing and Networking, Berkeley, CA, 1995, pp. 86-95.
[14] P. Jacquet, P. Muhlethaler, T. Clausen, A. Laouiti, A. Qayyum, L. Viennot, Optimized Link State Routing (OLSR) Protocol for ad hoc networks, IEEE INMIC, Pakistan, 2001.

[15] M. Gerla, Fisheye state routing protocol (FSR) for ad hoc networks, Internet Draft, draft-ietf-manet-aodv-03.txt, 2002.

[16] G. Pei, M. Gerla, T.W. Chen, "Fisheye State Routing: A Routing Scheme for Ad Hoc Wireless Networks", Proc. 2000 ICDCS Workshop on Wireless Networks and Mobile Computing, Taipei, Taiwan, Apr 2000, pp. D71D78

[17] T.W. Chen, M. Gerla, Global State Routing: a new routing scheme for ad-hoc wireless networks, in: Proceedings of theIEEE ICC, 1998.

[18] C. C. Chiang, T. C. Tsai, W. Liu, and M. Gerla, "Routing in clustered multihop, mobile wireless networks with fading channel," The Next Millennium, Proceedings of IEEE Singapore International Conference on Networks, SICON, pp. 197-221, 1997

[19] G. Pei, M. Gerla, X. Hong, and C. C. Chiang, "A wireless hierarchical routing protocol with group mobility," IEEE Wireless Communications and Networking Conference, WCNC '99, vol.3, pp. 1538 1542, New Orleans, LA, 1999.

[20] C. Toh, "A novel distributed routing protocol to support ad- hoc mobile computing", IEEE 15th Annual International Phoenix Conf., 1996, pp. 480-486.

[21] S. Das, C. Perkins, E. Royer, Ad hoc on demand distance vector (AODV) routing, Internet Draft, draft-ietfmanetaodv-11.txt, work in progress, 2002.

[22] C. E. Perkins and E. M. Royer, "Ad-hoc On-Demand Distance Vector Routing (AODV)", Proc. $2^{\text {nd }}$ IEEE Wkshp. Mobile Comp. Sys. And Apps., Feb 1999, pp. 9C100

[23] David B. Johnsson, David A. Maltz, Yih-Chun Hu, “ The Dynamic Source Routing (DSR) protocol for Mobile Ad Hoc Networks", Internet Draft, draft-ietf-manet-dsr10.txt, July 2004.

[24] R. V. Boppana and S. P. Konduru, "An adaptive distance vector routing algorithm for mobile ad hoc networks", Proc. IEEE Infocom 2001, April 2001, Volume 3, pp 1753-1762

[25]Douglas E. Comer, David L. Stevens, Internetworking with TCP/IP Vol-II, 3rd Ed., Pearson Education (Singapore), LPE, 2005

[26] Swapan K. Ray, Saurav Paira and Santanu Kr. Sen, "Modified Distance Vector Routing Avoids Count-ToInfinity Problem", Proc. International Conference CODIS 2004, held in Calcutta during Jan 8-10, 2004, pp 31-34.

[27] J. F. Kurose and K. W. Ross, Computer Networking: A Top-Down Approach Featuring the Internet, Third Edition, Pearson Education, Inc, Singapore, 2005

[28] S. K. Ray, J. Kumar, S. K. Sen and J. Nath, "Modified Distance Vector Routing Scheme for a MANET", Proc. of the 13th National Conference on Communications (NCC) held at IIT, Kanpur during Jan 26-28, 2007, pp. 197-201. 